\title{
A HEAT KERNEL CHARACTERIZATION OF ASYMPTOTIC HARMONICITY
}

\author{
FRANCOIS LEDRAPPIER
}

(Communicated by Peter Li)

\begin{abstract}
A compact negatively curved manifold is asymptotically harmonic if and only if the relation $4 \lambda_{1}=\beta$ holds, where $\lambda_{1}$ is the spectral gap of the Laplacian on the universal cover, and $\beta$ is the Kaimanovich entropy.
\end{abstract}

We consider a compact, connected boundaryless negatively curved Riemannian manifold. Such a manifold $M$ is called asymptotically harmonic if in the universal cover $\widetilde{M}$ of $M$ the mean curvature of the horospheres is constant. There are many equivalent formulations of this property [L2, Y]. They usually amount to saying that objects that are naturally associated to different features of the metric, such as the geodesic flow, the distance structure, or the heat kernel, do in fact coincide if and only if the manifold is asymptotically harmonic. Here we give another characterization that involves only asymptotic properties of the heat kernel on $\widetilde{M}$.

A beautiful recent result [FL] says that the geodesic flow of an asymptotically harmonic manifold is $C^{\infty}$-conjugate to the geodesic flow of a compact locally symmetric space of negative curvature. In particular, if $M$ is asymptotically harmonic, then its fundamental group $\Pi_{1}(M)$ is isomorphic to a cocompact discrete subgroup of isometries of a negatively curved symmetric space of the same dimension as $M$. By analogy with our result, one may ask for a random walk characterization of such groups. Note, however, that the most naive discretization of our result is not true [L4].

We denote $p_{t}(x, y)$ the heat kernel on $\widetilde{M}$ and consider the two following numbers:

$$
\begin{aligned}
& \lambda_{1}=\limsup _{t \rightarrow \infty}-\frac{1}{t} \ln p_{t}(x, x) \quad \text { for all } x \text { in } \widetilde{M}, \\
& \beta=\lim _{t \rightarrow \infty}-\frac{1}{t} \int p_{t}(x, y) \ln p_{t}(x, y) d y \quad[\mathrm{~K}] .
\end{aligned}
$$

In [L2] we showed that for any cover of a compact manifold $4 \lambda_{1} \leq \beta$ with equality in the case of the universal cover of an asymptotically harmonic manifold (see also Corollaries 3 and 4 below).

Received by the editors November 21, 1991.

1991 Mathematics Subject Classification. Primary 58G11.

Supported by SFB 256 (Bonn) and CNRS (Paris). 
Theorem 1. A compact negatively curved manifold is asymptotically harmonic if and only if $4 \lambda_{1}=\beta$.

Recall that $-\lambda_{1}, \lambda_{1}$ as defined above is the top of the spectrum of the Laplacian operator $\Delta$ in $L^{2}(\widetilde{M})$. Then the operator $\Delta-\lambda$ is coercive for $\lambda>-\lambda_{1}$ and the set of minimal positive $\lambda$-eigenfunctions of $\Delta$ can be represented as the boundary at infinity $\partial \widetilde{M}$ of the universal cover $\widetilde{M}$ [A]. For each $\xi$ in $\partial \widetilde{M}$, we denote $k_{\lambda}(x, y, \xi)$ the corresponding minimal positive $\lambda$-eigenfunction such that $k_{\lambda}(x, x, \xi)=1$. The function $k_{\lambda}$ is smooth in $x$ and $y$, and in particular the vector field $\bar{Y}_{\lambda, \xi}(x)=\Delta \ln k(x, \cdot, \xi)$ is a smooth vector field on $\widetilde{M}$ for any $\lambda, \xi$. Note also that from the proof of [A] if follows that $\bar{Y}_{\lambda, \xi}(x)$ depends continuously on $\xi$ (see [H, Lemma 3.2] for the case $\lambda=0$; the general case is analogous). Consider the unit tangent bundle $S \widetilde{M}$, and for $\tilde{v}$ in $S \widetilde{M},\left\{\gamma_{\tilde{v}}(t)\right.$, $t \in \mathbf{R}\}$ the geodesic in $\widetilde{M}$ defined by $\left(\gamma_{\tilde{v}}(0), \dot{\gamma}_{\tilde{v}}(0)\right)=\tilde{v}$. A leaf of the stable foliation of $S \widetilde{M}$ consists of all unit vectors $\tilde{v}$ with the same limit point in $\partial \widetilde{M}$ of $\gamma_{\tilde{v}}(t)$ as $t$ goes to $+\infty$. Each leaf is identified with $\widetilde{M}$ by the projection $\tilde{v} \rightarrow \gamma_{\tilde{v}}(0)$. For $\tilde{v}$ in $S \widetilde{M}$, define $\widetilde{Y}_{\lambda}(\tilde{v})$ as the unique vector in the tangent space to the stable leaf of $\tilde{v}$ which projects to $\widetilde{Y}_{\lambda, \gamma_{\hat{v}}(+\infty)}\left(\gamma_{\tilde{v}}(0)\right)$. The vector field $\widetilde{Y}_{\lambda}$ is a continuous vector field on $S \widetilde{M}$, tangent to the stable leaf, smooth along stable leaves and covariant under the action of $\Pi_{1}(M)$. It factorizes on $S M$ in a stable continuous vector field $Y_{\lambda}(v)$, smooth along stable leaves. Consider finally on the stable leaves of $S M$ the metric $g_{s}$ defined by lifting the metric on $M$ through the natural projection. We shall denote $w$ the unique probability measure on $S M$ that is harmonic for the stable foliation with $g_{s}$, i.e., that annihilates the leafwise Laplacian operator $\Delta^{s}$ defined by the metric $g_{s}$ (see $\left.[\mathrm{G}, \mathrm{L} 3, \mathrm{Y}]\right)$. We have the following formula:

Lemma 2. For all $\lambda>-\lambda_{1}, \beta+4 \lambda=\int\left\|2 Y_{\lambda}-Y_{0}\right\|_{g_{s}}^{2} d w$.

Proof of Lemma 2. We have, taking the leafwise divergence defined by $g_{s}$ :

$$
\operatorname{div}^{s} Y_{\lambda}(v)=\lambda-\left\|Y_{\lambda}(v)\right\|_{g_{s}}^{2} .
$$

We get Lemma 2 by integrating (1) with the measure $w$, using the "leafwise Green formula":

$$
\int \operatorname{div}^{s} Y(v) d w=-\int\left\langle Y, Y_{0}\right\rangle_{g_{s}} d w
$$

for any continuous stable vector field $Y$, smooth along the stable leaves [L3, Y] and the expression of $\beta$ as $\beta=\int\left\|Y_{0}\right\|_{g_{s}}^{2} d w[\mathrm{~K}]$.

Corollary 3. We have $\beta \geq 4 \lambda_{1}$.

Corollary 4. If $M$ is asymptotically harmonic, then $\beta=4 \lambda_{1}$.

Assume that the mean curvature of the horospheres is some constant $h$, and denote $\boldsymbol{\Theta}_{x, \xi}$ the Busemann function associated to a point $x$ in $\widetilde{M}$ and $\xi$ in $\partial \widetilde{M}$. For $\lambda>-h^{2} / 4$, the function

$$
k_{\lambda}(x, y, \xi)=\exp \left[\frac{h+\sqrt{h^{2}+4 \lambda}}{2} \Theta_{x, \xi}(y)\right]
$$

is a positive $\lambda$-eigenfunction and therefore $4 \lambda_{1} \geq h^{2}$. On the other hand, $\beta=\int\left\|Y_{0}\right\|^{2} d w=h^{2}$. 
Lemma 5. If $\beta=4 \lambda_{1}$, then for all $v$ in $S M,\left\|Y_{0}(v)\right\|_{g_{s}}^{2}=4 \lambda_{1}$.

Proof of Lemma 5. From Lemma 5 it follows that if $\beta=4 \lambda_{1}$, then $\int\left\|2 Y_{\lambda}-Y_{0}\right\|_{g_{s}}^{2} d w \rightarrow 0$ as $\lambda \searrow-\lambda_{1}$. We claim that we can find a sequence $\lambda_{n}, \lambda_{n} \searrow-\lambda_{1}$, such that for $w$-a.e. leaf, any compact $K$ on the leaf, we have

$$
\lim _{n \rightarrow \infty} \int_{K}\left\|2 \tilde{Y}_{\lambda_{n}}(\tilde{v})-\tilde{Y}_{0}(\tilde{v})\right\|_{g_{s}}^{2} d \operatorname{vol}_{g_{s}}(\tilde{v})=0 .
$$

To prove the claim write $\tilde{w}$ for the lifted measure from $w$ to $S \widetilde{M}$. The measure $\tilde{w}$ is locally equivalent, with continuous and positive density, to the product of the Lebesgue measure on the stable leaves and some measure $\mu^{\text {su }}$ on the transversals (see $[\mathrm{H}]$ ). Fix a compact piece of transversal $T$ of positive $\mu^{s u}$-measure and $R>0$. We can find a subsequence $\lambda_{n} \searrow-\lambda_{1}$, such that for $\mu^{s u}$-a.e. $\tilde{v}$ in $T$,

$$
\lim _{n \rightarrow \infty} \int_{B^{s}(\tilde{v}, R)}\left\|2 \tilde{Y}_{n}-\tilde{Y}_{0}\right\|_{s}^{2} d \mathrm{vol}=0 .
$$

Use a diagonal procedure to get the property for all $R$. We get that the property of the claim is true for a set of leaves of positive $\mu^{s u}$-measure on some transversal. By ergodicity of the leafwise harmonic measure $w$, such a set of leaves has full $w$-measure.

It follows from the claim that the function $\sqrt{k_{0}\left(\gamma_{\tilde{v}}(0), \cdot, \gamma_{\tilde{v}}(\infty)\right)}$ for $\tilde{w}$-a.e. $\tilde{v}$ is weakly approximated by $k_{\lambda_{n}}, n \rightarrow \infty$, in every $L^{2}$ of a bounded domain on the leaf of $\tilde{v}$. Therefore, for $\tilde{w}$-a.e. $\tilde{v}$, the above $\sqrt{k_{0}}$ is a weak $\left(-\lambda_{1}\right)$ eigenfunction of $\Delta$. Since $\sqrt{k_{0}}$ is in fact a smooth function, it follows that for $\tilde{w}$-a.e. $\tilde{v},\left\|Y_{0}(\tilde{v})\right\|_{g_{s}}^{2}=4 \lambda_{1}$. The lemma follows by continuity of $Y_{0}$ and $g_{s}$.

We now prove Theorem 1. Given Corollaries 3 and 4, we may assume that $\beta=4 \lambda_{1}$. By Lemma 5 we know that $\left\|Y_{0}\right\|^{2}=\beta$. We also know $[\mathrm{K}]$ that $\beta \leq h^{2}$, where $h$ is the topological entropy of the geodesic flow. Finally, we have for the measure $\mu$ of maximal entropy $h$ :

$$
h \leq \int X \log k_{0} d \mu=\int\left\langle X, Y_{0}\right\rangle d \mu \leq \sqrt{\beta},
$$

where $X$ is the geodesic spray (the first inequality holds for all probability measures invariant under the geodesic flow [L1]). All of these properties are compatible only if $Y_{0}=\sqrt{\beta} X \mu$-a.e. In that case, the function $k_{0}$ satisfies

$$
k_{0}(x, y, \xi)=\exp \left(\sqrt{\beta} \Theta_{x, \xi}(y)\right) \text { for } \mu \text {-a.e. }(x, \xi) \text {. }
$$

From $\Delta k_{0}=0$, it follows that the mean curvature of the horosphere at $v$ is $\sqrt{\beta}$ for $\mu$-a.e. $v$. This proves the theorem.

\section{BIBLIOGRAPHY}

[A] A. Ancona, Negatively curved manifolds, elliptic operators and the Martin boundary, Ann. of Math. (2) 125 (1987), 495-536.

[FL] P. Foulon and F. Labourie, Sur les varietés compactes asymptotiquement harmoniques, Invent. Math. (to appear).

[G] L. Garnett, Foliations, the ergodic theorem and Brownian motion, J. Funct. Anal. 51 (1983), 283-311.

[H] U. Hamenstädt, An explicit description of the harmonic measure, Math. Z. 205 (1990), 287-299. 
[K] V. A. Kaimanovich, Brownian motion and harmonic functions on covering manifolds. An entropy approach, Soviet Math. Dokl. 33 (1986), 812-816.

[L1] F. Ledrappier, Ergodic properties of Brownian motion on covers of compact negatively-curved manifolds, Bol. Soc. Brasil. Mat. 19 (1988), 115-140.

[L2] _ - Harmonic measures and Bowen-Margulis measures, Israel J. Math. 71 (1990), 275287.

[L3] _ Ergodic properties of the stable foliations, Ergodic Theory and Related Topics III (Proceedings, Güstrow 1990), Lecture Notes in Math., vol. 1514, Springer-Verlag, Berlin and New York, 1992, pp. 131-145.

[L4] _ Sharp estimates for the entropy, Harmonic Analysis and Discrete Potential Theory (M. Picardello, ed.), Plenum Press, New York, 1992, pp. 281-288.

[Y] C. B. Yue, Integral formulas for the Laplacian along the unstable foliation and applications to rigidity problems for manifolds of negative curvature, Ergodic Theory Dynamical Systems 11 (1991), 803-819.

Laboratoire de Probabilité, Tour 56, Université Paris Vi, 4 Place Jussieu, 75252 Paris Cedex 05, France 